\title{
INFLUENCE OF SAMPLE-MOVEMENT ON PARTICLE-RANDOMIZATION OF POWDER SAMPLES IN THE PARALLEL-BEAM GEOMETRY
}

\author{
K. BURGER and J. IHRINGER \\ Institut für Kristallographie der Universität Tübingen, Charlottenstraße 33, \\ D-72070 Tübingen, Germany
}

(Received 14 February 1995)

\begin{abstract}
Today, from powder X-ray diffraction the scientists want to obtain high resolution diffraction patterns with reliable Bragg-reflection intensities. Two well-known and closely connected obstacles on the way are texture and particle randomization of the sample, which strongly influence the measured intensities. In the work presented here, we examine the second problem.

Especially with high resolution diffractometers, for well crystallized or highly absorbing samples the number of contributing crystals in the powder is too small, thus introducing significant errors in the profiles and measured intensities of Bragg-reflections. This may be the major source of inaccuracy in data used for structure determination. Calculations of the error of the integrated intensities are presented, for the high resolution, parallel beam geometry at a synchrotron X-ray source. Results exhibit errors of $40 \%$ in the range of highest resolution for a sample of $3 \mu \mathrm{m}$ crystals of corundum, with the sample at rest. To enhance randomization, several methods of sample-movement are considered. A new effective method is proposed, where the rotation axis of the flat sample-disk is slightly inclined out of the diffraction plane.
\end{abstract}

KEY WORDS: Power diffraction sample movement particle randomization parallel beam geometry.

\section{INTRODUCTION}

For high resolution powder diffraction, well collimated X-ray beams (divergence $<0.5$ mrad) and a narrow bandwidth monochromatization $\left(\Delta \lambda / \lambda<0.5 \cdot 10^{-3}\right)$ have to be used-yet inevitably connected with this is the problem of particle randomization of the powder.

In a powder sample, only a very small fraction of the particles exposed to the $\mathrm{X}$-ray beam is oriented correctly for reflection towards the detector, roughly one particle in a million. Therefore, if the size of the crystals is too large, or the diffracting volume is too small, a random orientation distribution of the powder is not the case any more. Thus, an essential assumption for the derivation of the generally used formulas for the reflectivity of powder samples (Zachariasen, 1945), is not valid any more. This insufficient randomization is the origin of the spottiness sometimes observed on Debye Scherrer-films, and an analogue effect is occasionally seen in the form of saw-toothed profiles in the synchrotron $\mathrm{X}$-ray patterns from highly crystallized materials. The importance of rotating or oscillating the samples has already been emphasized; however, in spite of some sample movement during the measurements, insufficient randomization may be the major factor contributing to an unexpectedly poor intensity fit for a 
well-characterized and highly crystalline material (see Parrish et. al., 1986, and Cox, 1992). In our work (Burger, 1993), by X-ray-optical and statistical considerations, we have calculated the error for the sample at rest, using an approximation somewhat analogous to the well known formalism of Caglioti. We then considered the influence of sample movement, of which several methods are discussed here: simple rotation of the flat sample around it's surface normal, oszillation about the $\omega$-axis perpendicular to the diffraction plane, and two new methods of precession of the reciprocal lattice vectors around the vector which bisects the scattering angle. As an example, the parameters of the instrument 'B2' at HASYLAB, DESY (Hamburg, Germany) are used in the calculations. A new effective method of sample-movement is proposed: the 'precession of Bragg-planes'.

\section{THEORY}

Given the diffractometer's geometry, sample absorption and particle size, the path of rays in the instrument and the number of reflecting crystals can be calculated.

\section{Geometrical considerations:}

The calculations are made for experiments at diffractometer 'B2' at HASYLAB, which uses the parallel beam geometry with double crystal monochromator, a flat sample disk and an analyser crystal as 'receiving slit'. For a description of this type of diffractometer see Cox et. al. (1983), and Hastings et. al. (1984). Usually, measurements are carried out in the way of a $\theta-2 \theta$-scan (compare figure 1). With an analyser crystal used, there is a big advantage in the absence of errors of the displacement type, i.e. a possibly inaccurate position of the sample induces no errors in the measurement, as they are known with focusing geometries (e.g. Guinier geometry). This opens up new possibilities for the sample movement during the measurement.

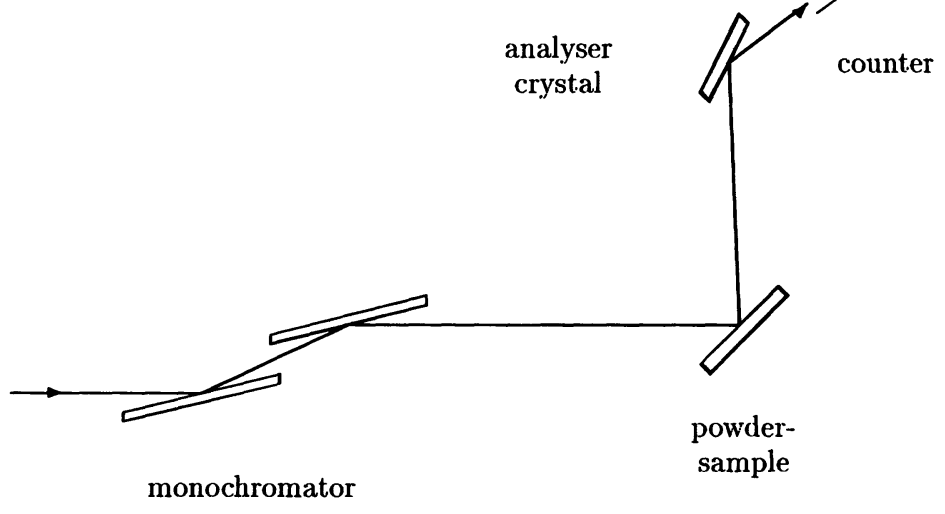

Figure 1 Path of rays of the diffraktometer 'B2' at HASYLAB, DESY, Hamburg. The primary $X$ ray beam incidents horicontally on a double monochoro-mator, and is then symmetrically reflected by the sample-disk at scattering angle $2 \theta$. 


\section{Reflection of one powder crystal}

In a simple model, the powder particles are assumed to be small perfect crystals of similar size and shape, distributed randomly within the powder. Within the sample the radiation is weakened by absorption with coefficient $\mu$. To reflect into a given Debye-Scherrer cone (hkl), the Bragg-planes have to be oriented very precisely near the 'ideal' Bragg-angle $\theta=\theta_{h k l}$, since the beam is very well collimated. Then the integral intensity $I$ of one crystal depends on it's location $x$ under the sample's surface and on it's deviation $\eta$ from the Bragg-angle $\theta$, according to

$$
I(x, \eta)=I_{1} \cdot e^{-2 \mu x} \mathrm{e}^{-\mathrm{B} \eta^{2}}
$$

$I_{1}$ is a constant proportional to the kinematical reflectivity of a single crystal. The first exponential term describes the absorption of the incident and reflected radiation within the sample (symmetric reflection). The second exponential term describes the fraction of incident radiation matching the crystal's misorientation $\eta$. This fraction can be approximated by a Gaussian function $N(\eta)$ with parameter $B$, in analogy to the well-known formalism of Caglioti (1958, and 1962). Since the crystals are assumed to be well crystallized (no mosaic spread), their angular range of reflection can be neglected compared with the halfwidth $\eta_{1 / 2} \sim 1 / \sqrt{B}$. In the further treatment, the irradiation was assumed to be constant on the whole surface exposed, i.e. the spectral fanning-out of the radiation behind the monochromator was neglected.

\section{Use of Caglioti's formalism to find $N(\eta)$}

This formalism is often used to calculate the resolution of $\mathrm{X}$-ray or neutron diffractometers. The divergence of the incoming beam and all optical components (monochromator crystals, slits etc.) of the instrument are represented by Gaussian functions describing their reflectivities for photons (or neutrons) deviating from the ideal path in the instrument. Even if individual functions cannot be well approximated as Gaussian functions, the resulting convolution of several different functions can. In the calculation, the path and probability of transit through the instrument are calculated for a photon with some starting parameters, which is incident in a 'white' beam of angular divergence $\alpha$. Finally, the outcoming intensity distribution is integrated over all possible starting parameters to get the resolution function of the instrument. For the instrument used at HASYLAB, this task has been carried out by Reinhardt (1989). See also Wroblewski (1988). The sample is treated as ideally reflecting.

This formalism can be used in a modified way to calculate $N(\eta)$. One follows the path of the photons to the sample, then integrates all photons incoming with the correct parameters to be reflected at a given Bragg-plane with orientation angle $\eta$. By elementary calculations one arrives at the result

$$
\begin{aligned}
N(\eta) & =A \cdot \mathrm{e}^{-\mathrm{B} \eta^{2}} \\
\mathbf{B} & =\frac{4 \ln 2}{a^{2} \Delta_{M}^{2}}\left(2-\frac{(2-2 / a)^{2}}{\Delta_{M}^{2} / \alpha^{2}+2(1-1 / a)^{2}}\right) \\
\eta_{1 / 2} & =2 \sqrt{\ln 2} / \sqrt{B}
\end{aligned}
$$

with $A$ a scaling factor, $a \equiv \tan \theta / \tan \theta_{M}$. $\theta$ is the Bragg angle at the sample, $\theta_{M}$ and $\Delta_{M}$ are the monochromator crystals' Bragg angle and mosaic-spread. In this way, 
$\eta_{1 / 2}$ depends on the diffractometer's parameters and on the reflection angle $\theta$. It describes the angular range in which a crystal has to be oriented (see figure 2) and is very small especially near the monochromatorangle $\left(\theta \approx \theta_{M}\right)$

Number of possibilities for reflection in the powder

With symmetrical incidence and reflection of the radiation, the reflecting powder volume is independent of the scattering angle $2 \theta$. It can be calculated from the cross-section of the incident beam $A_{s}$ and the absorption of the powder $\mu$. Within one extinction-length $t=1 / \mu$ the total number of crystals is $N=A_{s} t / \delta V$, with $\delta V=\pi D^{3} / 6$ the volume of one crystal of diameter $D$. The multiplicity $j_{H}$ has to be considered, also the probability $\sim \cos \theta$ of finding crystals oriented at $\theta$. Finally, the detector measures only a small fraction of the total scattered radiation due to the detector's fixed angle of acceptance $\alpha_{z} \approx 10 \mathrm{mrad}$ perpendicular to the vertical scattering plane. The number of reflections found at a certain position $x$ and misorientation angle $\eta$ within the powder is

$$
\rho d x d \eta=\frac{\alpha_{t} A j_{H}}{(8 \pi \sin \theta) \delta \mathrm{V}} d x d \eta
$$

\section{Halfwidth of $N(\eta)$-function}

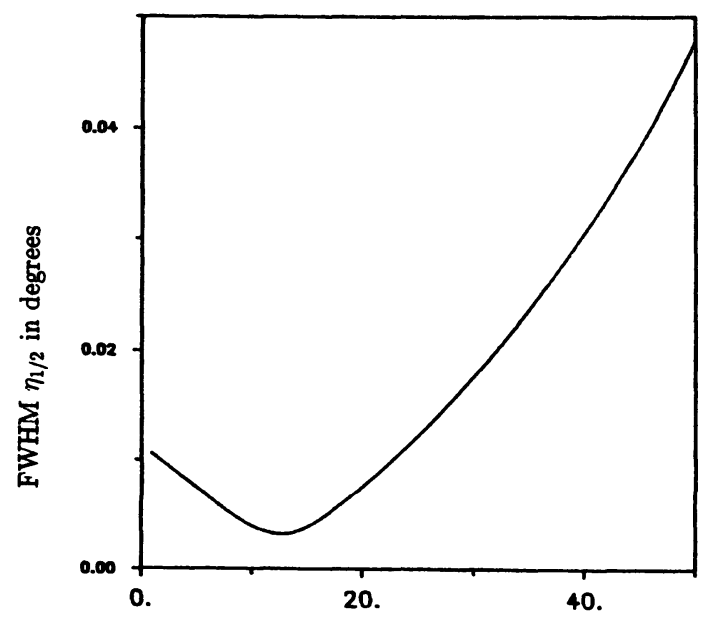

Bragg angle $\theta$

Figure 2 The dependence of the halfwidth $\eta_{1 / 2}$ of the function $N(\eta)$ on the power reflection's scatteringangle $2 \theta$. The shown values are for the parallel-beam-geometry at diffractometer 'B2' at HASYLAB, DESY (Hamburg, Germany) with two germanium crystals' (111)-reflection used in the double monochromator. The divergence of the primary beam is $\alpha \approx 0.2$ mrad. The monochromator crystals' mosaic-spread is $\Delta_{M} \approx 0.08 \mathrm{mrad}$. At wavelength $\lambda=1.54 \AA$ the monochromator angle is $\theta_{M}=13.6^{\circ}$. Near $\theta_{M}$ the function $N(\eta)$ has it's minimum halfwidth. 


\section{Statistical considerations}

From the formulas given above, one can calculate the mean and variance of one reflection's integral intensity measured. $x$ and $\eta$ are treated as random variables with equipartition distribution, i.e. no correlation of the crystals is allowed for. We obtained the relative error for the sample at rest $I_{H} \equiv I_{\text {hlt }}$,

$$
\Delta I_{H} / I_{H}=\sqrt{\frac{\sqrt{21 \mathrm{n} 2}}{\sqrt{\pi}} \frac{\mu}{\rho \cdot \eta_{1 / 2}}} \sim \sqrt{1 / n}
$$

$n \equiv \rho \cdot \eta_{1 / 2} \cdot t$ is the approximate mean number of crystals contributing substantially for the sample at rest. (see figure 3). The resulting error can be found in figure 4 (continuous line).

\section{Number of reflecting crystals}

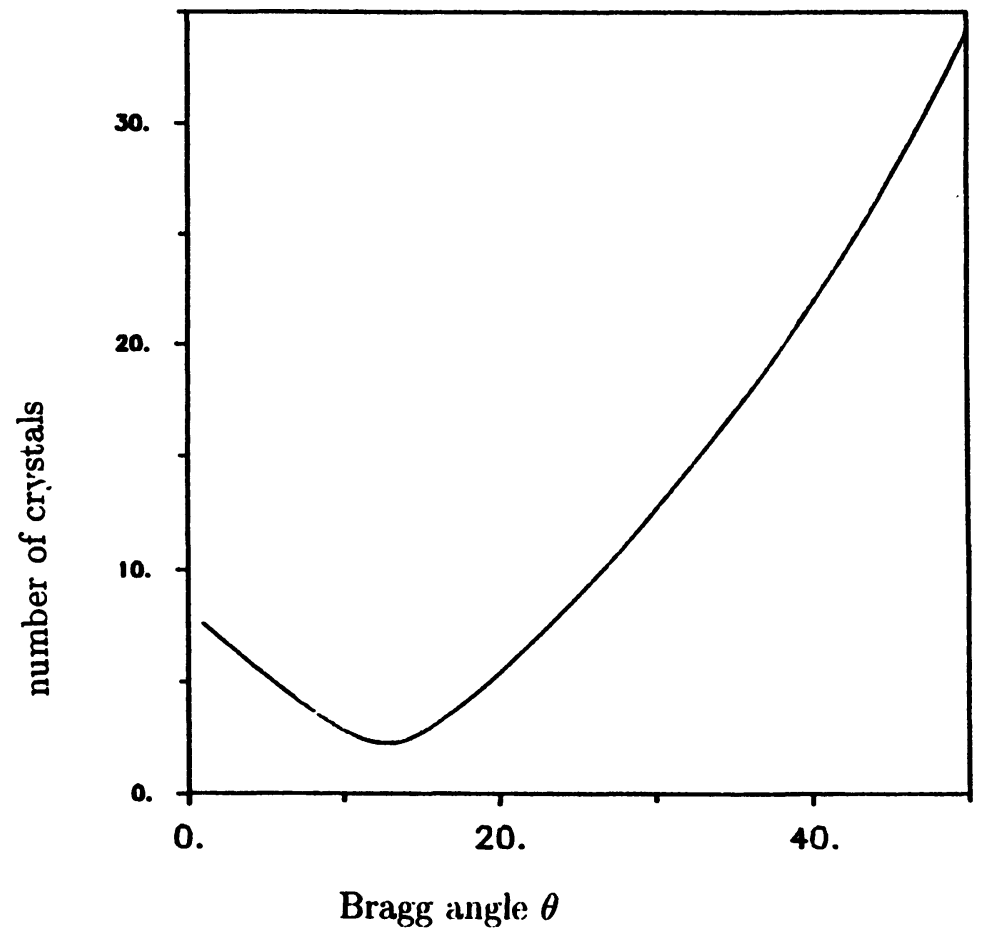

Figure 3 For a $\alpha-\mathrm{Al}_{2} \mathrm{O}_{3}$ corundum sample with crystal size $D=3 \mu \mathrm{m}$ the calculated mean effective number of reflecting crystals is shown. In the region of the monochromator angle $\theta_{m}$, less than five particles determine the measured intensities to a high degree. 


\section{Error of integral intensity}

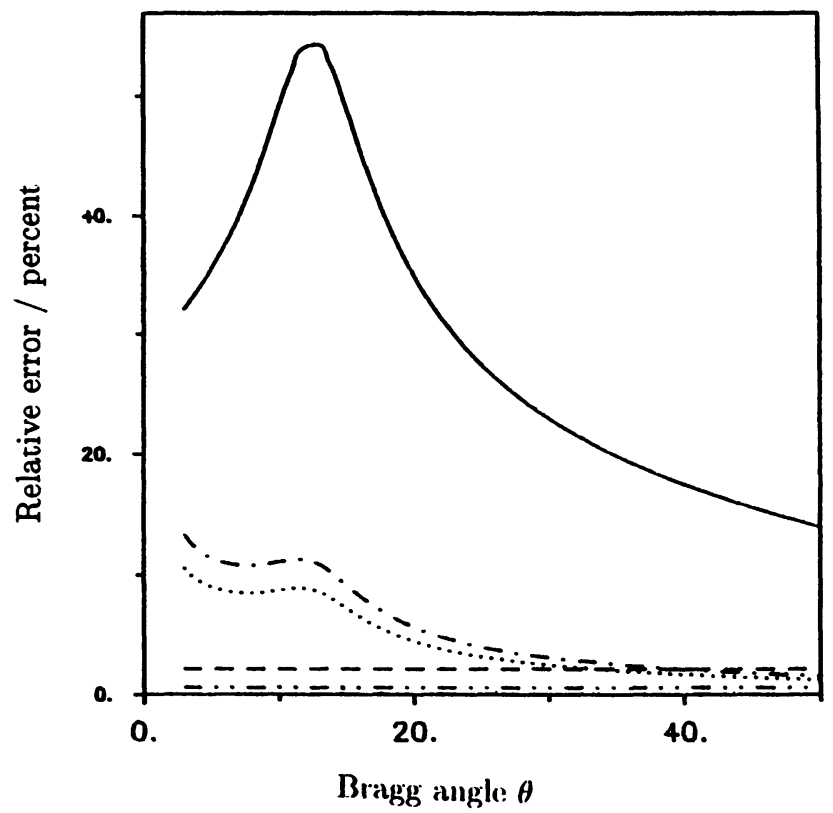

Figure 4 Theoretically calculated relative errors of the measured intensity of a reflection at scattering angle $2 \theta$ of the powder sample. This error is solely from the inadequate randomization of the power. As an example, a powder of ideal corundum crystals $\alpha-\mathrm{Al}_{2} \mathrm{O}_{3}$ of diameter $D=3 \mu \mathrm{m}$ and absorption $\mu=120 / \mathrm{cm}$ is assumed. For larger crystals the error grows proportional to $D^{3 / 2}$, i.e. for $D=30 \mu \mathrm{m}$ the errors are about 32 times as large.

Shown are the errors for the stationary sample (continuous line), for the sample rotating in it's plane (dotted line), for 'precession of Bragg-planes (1)' (broken and dotted line), for 'oszillation in $\omega$ ' (broken line) and for the 'precession (2)' (broken and double dotted) $(\lambda=1.54 \AA)$.

\section{Influence of sample movement}

A movement (e.g. rotation) of the sample disk during measurement gives more powder particles the opportunity to contribute to the measured reflection. This doesn't change the mean intensities, but it reduces the error which has to be expected solely from the inadequate randomization of the powder sample. For the methods considered here, the sample movement brings all crystals into the reflection position for about the same duration. It follows that the movement can be taken into account by multiplying the density of reflections $\rho$ by a sample-movement-factor $f_{r}$, if $f_{r}$ more crystals come to participate because of the movement. By this factor the different movements can be compared easily. 


\section{SOME METHODS OF SAMPLE-MOVEMENT}

For a theoretical treatment the different kinds of sample-movement can be divided into 1. 'randomization in real space', where the irradiated surface of the sample is enlarged, 2. 'randomization in reciprocal space', where the sample's orientation is changed, and 3. 'combined methods'. Several methods for different geometries have been already mentioned, see e.g. Klug $\mathcal{E}$ Alexander pp. 193-197, Parrish $\mathcal{E}$ Hart (1986).

In the following comparison the parameters of the powder diffractometer ' $\mathrm{B} 2$ ' at HASYLAB (DESY, Hamburg, Germany) are used, with beam-divergence $\alpha \approx 0.2 \mathrm{mrad}$ and mosaic-spread of monochromator Ge-(111) crystals $\Delta_{M} \approx 0.08 \mathrm{mrad}$. At the scattering angle $2 \theta=40^{\circ}$ and wavelength $\lambda=1.54 \AA$, the offering-function's halfwidth is $\eta_{1 / 2}=7.6 \cdot 10^{-3}$ degrees. With this parameters, $f_{r}$ can be calculated to compare the methods. The higher the factor $f_{r}$, the smaller the error of the measured intensities $I_{H}$ due to insufficient randomization of the powder-sample, i.e. $\Delta I_{H} / I_{H} \sim 1 / \sqrt{f_{r}}$. Several methods were chosen-the following method 1 . is an example of the 'randomization in real space', methods 2.-4. are examples of 'randomization in reciprocal space'. Not only particle randomization, but also texture averaging is different.

1. Rotation: On the rotating sample-disk the beam irradiates a ring-sllaped area of radius $R$, since the beam hits the sample at a horizontal distance $R$ away from the center (see figure 5). The beam's cross section can be approximated by a rectangle of height $h_{s} \approx 0.5 \mathrm{~mm}$ and width $b_{s} \approx 2 \mathrm{~mm}$. The diameter of the sample disk is 53 $\mathrm{mm}$ for measurements at room temperature, and $37 \mathrm{~mm}$ for low temperature experiments. The factor $f_{r}$ is the ratio of irradiated area due to the rotation and area in the stationary case, namely

$$
f_{r}=2 \pi R \frac{\sin \theta}{h_{s}}
$$

With $R=14 \mathrm{~mm}$ and $h_{s}$ and $\theta$ given,

$$
f_{r}=180 \sin \theta=60
$$
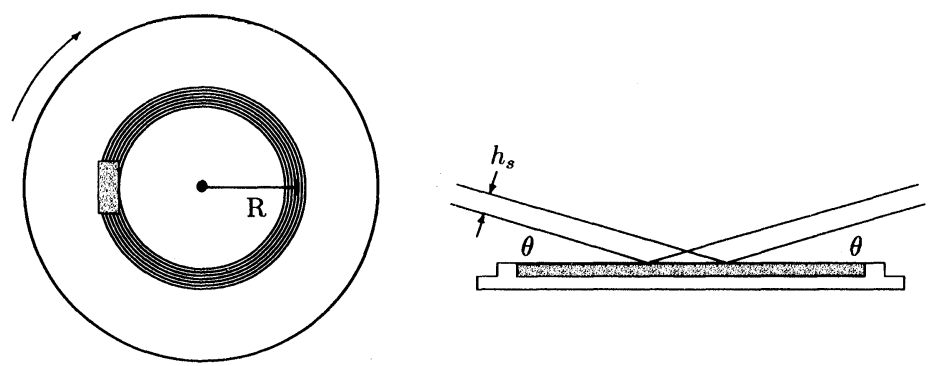

Figure 5 First method of sample movement: Rotation of the sample-disk. Left: View of the sample's surface. The shaded rectangle marks the momentanously irradiated area of the sample, the total irradiated area is a ring with radius $R$ due to the rotation. Right: The sample seen from the side with incident and reflected beam; section in the vertical scattering plane. 
In that case, about sixty times more crystals can participate at the measurement due to the sample's rotation in it's plane. Reflecting Bragg-planes are oriented parallel to the sample's surface. In a low-temperature measurement, $R$ cannot be larger than several millimeters due to experimental limitations; in this case this movement is of very limited use, and often the next method is used instead.

2. Oszillation in $\omega$ : The beam hits the sample's center. The sample doesn't rotate, instead it is oszillating in $\omega$ around the position of symmetric incidence and reflection $\omega_{0}=\theta$. With the maximum tilt-angle called $\Delta \omega, \omega$ varies between $\theta+\Delta \omega / 2$ and $\theta$ $-\Delta \omega / 2$ (see figure 6).

During the movement, the irradiated area is nearly constant. The halfwidth $\eta_{1 / 2}$ is a measure for the 'width of the receiving slit'. If, during the measurement of one data point, the sample is moved through the angle $\Delta \omega$ with constant velocity, the factor $f_{r}$ becomes

$$
f_{r}=\Delta \omega / \eta_{1 / 2}
$$

$\eta_{1 / 2}$ depends strongly on the scattering angle $2 \theta$ of the sample and is very small near the region of the diffractometer's maximum resolution. Nevertheless, as long as $\eta_{1 / 2}$ $\ll \Delta \omega$ for $2 \theta<150^{\circ}$, the relative error $\theta I_{H} / I_{H}$ is independent of $\theta$ (see formula (6)).

At instrument B2, this type of movement is performed by rotating the $\omega$-circle, with the sample-holder mounted at the center. The continuous movement during the measurement is easily performed since it is a built-in option of the controlling software, but it is timeconsuming and puts a certain mechanical strain on the $\omega$-circle, especially when the heavy cooling-device is mounted. The velocity is limited to $0.5^{\circ}$ per second, so that $\Delta \omega \leq 2^{\circ}$. With this movement, the factor $f_{r}$ is

$$
f_{r}=265
$$

$\left(f_{r}=600\right.$ at $\left.\theta=\theta_{M}=13.6^{\circ}\right)$.

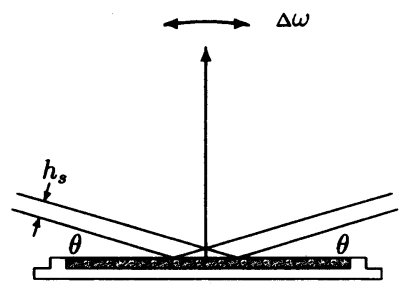

\section{In RECIPROCAL SPACE}

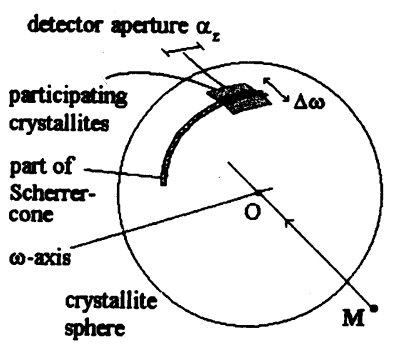

Figure 6 Method 2: Oszillation in $\omega$. Left: The sample oszillates around the angle $\omega_{0}=\theta$ of symmetric incidence and reflection. Section through the sample-disk in the plane of diffraction. Right: This sample movement brings the crystals in the shaded area to participation. 
3. Precession of Bragg-planes (1): As in 2., the beam hits the center of the sample. The sample is tilted by a fixed angle of $\Delta \omega$ away from the symmetric orientation, $\omega$ $=\theta+\Delta \omega$. The sample is rotating about its surface normal as in the first method. But now the reflecting Bragg-planes are tilted by $\Delta \omega$ against the surface and not parallel as in the first method. Thus the normals of the participating crystals precess around the normal of the sample's surface. The effect can he seen clearly in Ewald's construction in reciprocal space (see figure 7).

The factor $f_{r}$ can he received considering the 'width of the receiving slit' $\eta_{1 / 2}$ within the scattering plane and the axial 'length of the receiving slit' $\alpha_{z}$,

$$
f_{r}=4 \pi(\sin \theta) \frac{\Delta \omega}{\alpha_{z}}
$$

With $\Delta \omega=2^{\circ}$ assumed,

$$
f_{r}=15
$$

The reason for this disappointing result is, that the different reflecting crystals' normal vectors move parallel to the 'length of the receiving slit' during the sample's rotation. A remedy is possible by a slight modification:

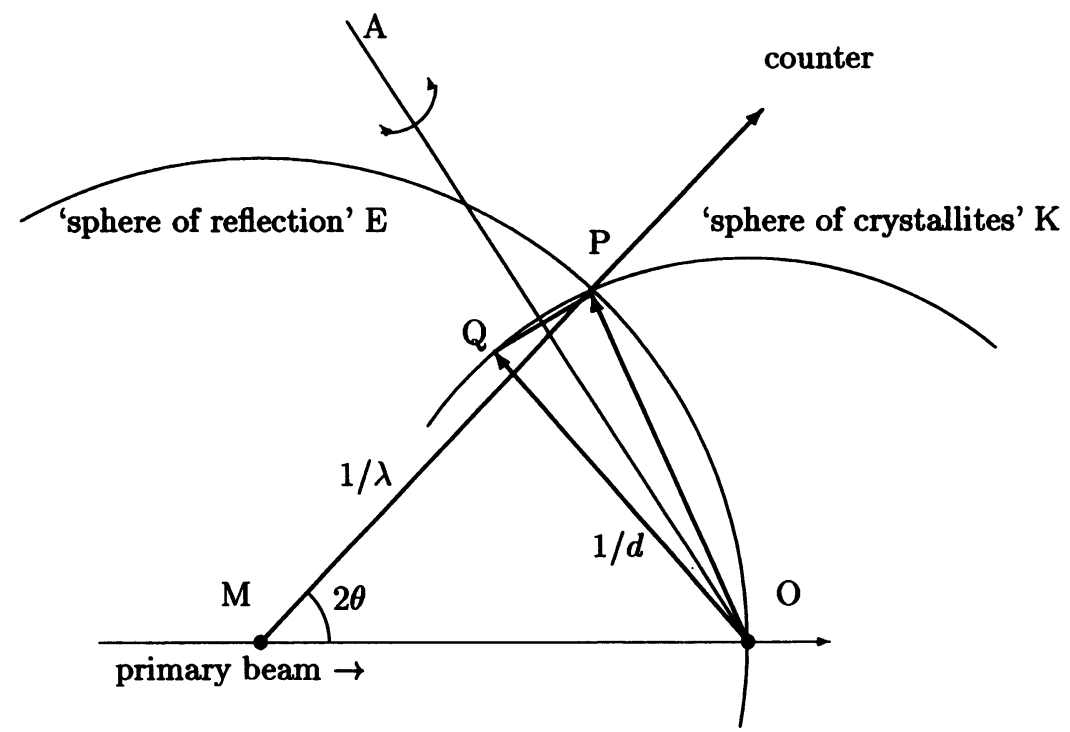

Figure 7 Method 3: Precession of Bragg-planes (1). Scetch of the situation in the reciprocal space in the plane of diffraction. Reflecting crystallites are oriented near point $P$. Because of the rotation of the 'misoriented' sample-disk about it's surface-normal $A$, a ring on $K$ with diameter (QP) contains crystals participating at the reflection. 
4. Precession of Bragg-planes (2): The beam hits the center of the rotating sample, as before. But now the sample's surface-normal is tilted out of the scattering plane by a certain angle $\emptyset$, i.e. the sample is tilted around the axis of the incoming beam, while the sample's surface is still in the symmetric orientation $\omega=\theta$. Now the participating crystals' normal vectors cross the 'receiving slit' in reciprocal space perpendicularly (see figure 8), which gives a much higher 'flux' during the rotation, namely the factor

$$
f_{r}=2 \pi \frac{\phi}{\eta_{1 / 2}}
$$

Also, the $\theta-2 \theta$-coupling of sample orientation and detector position has not to be changed. With $\emptyset=6^{\circ}$ assumed, one gets

$$
f_{r}=5000
$$

Here, as in method 2. oszillation in $\omega)$, this factor depends strongly on $\theta\left(f_{r}=11200\right.$ at $\left.\theta=\theta_{M}=13.6^{\circ}\right)$, but $\Delta I_{H} / I_{H}$ is constant. The high factor is received on small mechanical expense, since only the sample-disk is rotating, driven by a small extra motor which is already installed. It is of no harm when the beam does not hit the sample-disk's center exactly.

Methods 2. and 4. have the advantage that they give very good results in the "critical region' near the monochromator angle $\theta_{M}$, where only very few crystals reflect without movement. Also the relative error $\Delta_{H} / I_{H}$ is constant for all scattering angles. Method 4. seems to he the best, as it gives theoretically a very high improvement of precision of the intensity measurements. For the example of a corundum powder $\alpha-\mathrm{Al}_{2} \mathrm{O}_{3}$, consisting of ideal crystals of diameter $D=3 \mu \mathrm{m}$, the relative errors to he expected according to our calculations are shown in figure 4.
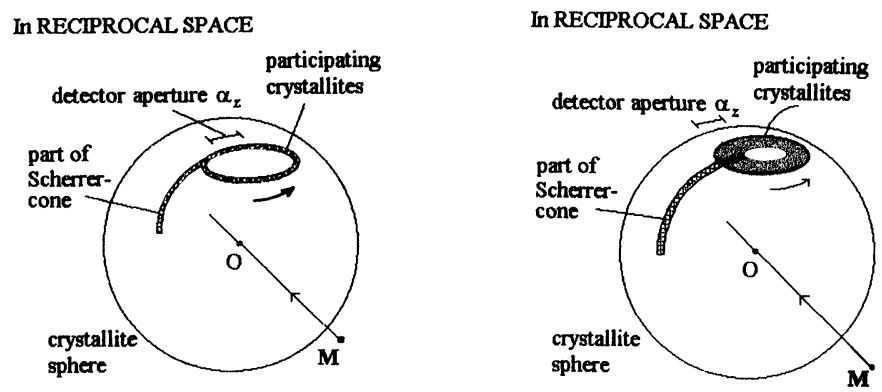

Figure 8 Methods 3 and 4: 'Precession of Bragg-planes' (1) and (2). Left: 'Precession (1)'. The rotation axis of the sample disk its tilted within the diffraction plane, away from its ideal symmetrical position $\omega=\theta$. The small part of the Scherrer-cone marked dark describes the momentanously reflecting crystals. It length is $\alpha_{z}$ and its width is $\eta_{1,2}$. Due to the rotation, the participating crystals form a ring on the surface of the crystallite sphere, whose radius is given by the tilting-angle and whose thickness ist given by $\eta_{1 / 2}$.

Right: Analogue situation for the tilting out of the diffraction plane. Now the thickness of the ring of participating crystals is given by $\alpha_{z} \gg \eta_{1 / 2}$, thus leading to a much better randomization of the power sample. 


\section{CONCLUSION}

For structure analysis the precise determination not only of the positions but also of the integrated intensities of Bragg-reflections is essential. Insufficient randomization of the powder has a marked influence: because of the use of highly monochromatic and well collimated X-ray beams, only very few particles can contribute to the measurement. The result is a large error in the intensities measured, if not some sample-movement is applied. Witll the help of an approximation analogous to the well known formalism of Caglioti, the errors of the integrated intensities have been calculated for the parallelbeam geometry, and remedies in form of several types of movements have been compared.

The unsufficient randomization is critical in high resolution measurements of well crystallized samples with sizes larger than one micrometer or with significant absorption. The region of Bragg-angles $\theta$ near the monochromator angle $\theta_{M}$ yields the most problems. The profiles are ill-formed and the intensities are deviating from theory.

Four different methods are compared: these involve 'randomization in real space' and 'randomization in reciprocal space'. Texture averaging is different depending on the method. An effective new method has been proposed, the 'precession of Bragg-planes', where the sample-disk is tilted by a fixed small angle around the incident beam and the disk is rotating in it's plane during measurement.

\section{References}

Burger, K., Masters thesis, Tübingen 1993.

Caglioti, G., A. Paoletti und F. P. Ricci, Nucl. Instr., 3 (1958) 223.

Caglioti, G. (1962), Effect of Collimators on Resolution and Luminosity of Diffraction Patterns, Adv. Course on Neutr. Crys. Spectr. Kjeller, Norway (Juni 1962).

Cox, D. E., J. B. Hastings, W. Thomlinson and C.T Prewitt (1983), Nucl. Instr. Meth., 208, 573578.

Cox, D. E., in Synchrotron Radiation Crystallography (Ph. Coppens ed.), p. 227 (1992).

Hastings, J. B, W. Thomlinson and D. E. Cox (1984), Synchrotron X-ray Powder Diffraction, J. Appl. Cryst., 17, 85-95.

Klug, H. P., und L. E. Alexander, X-Ray Diffraction Procedures, Wiley, New York 1967.

Parrish, W., M. Hart and T. C. Huang, Synchrotron X-ray Polycrystalline Diffractometry, J. Appl. Cryst., (1986) 19, 92101.

Reinhardt, J., Masters thesis, Tübingen 1989.

Wroblewski, Th., J. Ihringer and J. Maichle, Nucl. Inst. Meth., (1988), A266, 664-667.

Zachariasen, W. H., Theory of X-Ray Diffraction it Crystals, New York 1945. 